\title{
UHRF1: a novel metabolic guardian restricting AMPK activity
}

Che-Chia Hsu ${ }^{1,2}$, Yau-Sheng Tsai ${ }^{1,2}$ and Hui-Kuan Lin (D) ${ }^{1 凶}$

(c) CEMCS, CAS 2021

Cell Research (2022) 32:3-4; https://doi.org/10.1038/s41422-021-00589-4

\begin{abstract}
AMP-activated protein kinase (AMPK) serves as a central guardian maintaining energy homeostasis under nutrient stress and/or energy crisis. The study by $\mathrm{Xu}$ et al. identified that a nuclear factor UHRF1 (ubiquitin-like with plant homeodomain and RING finger domains 1) suppresses AMPK activity in the nucleus by recruiting PP2A to dephosphorylate AMPK at T172, which in turn governs cytoplasmic AMPK activity in regulation of glucose and lipid metabolism.
\end{abstract}

Eukaryotic cells operate the intrinsic mechanism to sense the imbalance of energy through activation of AMP-activated protein kinase (AMPK), which serves as a guardian to regulate diverse signal and metabolic pathways in response to various environmental insults. AMPK serves as an energy sensor to maintain energy homeostasis and cell survival by phosphorylating several key metabolic proteins involved in reprograming the cellular metabolism. ${ }^{1}$ AMPK activation in response to distinct stresses triggers catabolic metabolism, but turns off anabolic metabolism. ${ }^{1}$ To comprehensively understand the mechanism of how AMPK activation is modulated upon energy stress is pivotal for offering the insight and strategy into the treatment for metabolic diseases and cancer. ${ }^{1}$

AMPK is activated by two upstream kinases, LKB1 and CAMKK2, both of which phosphorylate AMPKa at T172, thereby triggering AMPK activation. In contrast, the activity of AMPK is attenuated by PP2A-mediated AMPK dephosphorylation at T172. Interestingly, AMPK activation is directly orchestrated by metabolic fluctuation like increase of AMP/ATP ratio under energy stress. ${ }^{1}$ AMP serves as a direct allosteric activator of AMPK complex, whereas ATP acts as an inhibitor of AMPK complex through their direct binding to AMPKy subunit. ${ }^{1}$ Notably, recent studies revealed that specific metabolites derived from glucose metabolism modulate AMPK activity and their biological functions have been identified. For instance, fructose-1,6-biphosphate (FBP) binds to aldolase and leads to AMPK inactivation by disrupting AXIN/LKB1/v-ATPase/ Regulator complex under the glucose proficient condition. ${ }^{2}$ Moreover, inositol derived from glucose-6-phosphate (G6P) acts as an allosteric inhibitor of AMPK by directly binding to AMPKY subunit and competes with AMP for AMPKy binding leading to restriction of AMPK-mediated mitochondrial fission. ${ }^{3}$ Thus, inositol/AMP ratio also critically impacts AMPK activation. It is important to note that subcellular localization of AMPK at different cellular compartments is another key mechanism to regulate AMPK activation by different stimuli. ${ }^{1,4}$ Of note, AMPKa2 subunit with the nuclear localization signal (NLS) is subjected to the nucleocytoplasmic translocation and in turn phosphorylates transcriptional factors in the nucleus, thereby affecting nuclear epigenetic landscapes. ${ }^{5}$ While diverse regulatory mechanisms for AMPK activation described above have been unraveled, how AMPK activation is turned off in the nucleus and how PP2A phosphatase is engaged to suppress AMPK activity under energy stress are currently unknown.

The study published by Wong and his colleagues offers the critical answers to these key questions. They uncovered that AMPK activity in the nucleus is terminated by nuclear factor UHRF1 (ubiquitin-like with plant homeodomain and RING finger domains 1), which has been shown to govern the balance of multiple epigenetic pathways (Fig. 1). ${ }^{6}$ UHRF1, an E3 ligase that is overexpressed in numerous human cancers, acts as an epigenetic regulator essential for DNA methylation by promoting DNMT3A ubiquitination and degradation. UHRF1 functions as an oncogene that drives tumor growth by regulating DNA hypomethylation. ${ }^{7-9}$ This study identifies UHRF1 as a negative regulator of AMPK in diverse cell models. Surprisingly, UHRF1 mediates AMPK inactivation independently of AMPK upstream kinases as well as E3 ligase activity of UHRF1 and its regulation on DNA methylation and DNA damage repair. ${ }^{10}$ Instead, UHRF1 directly binds to AMPK and recruits the phosphatase PP2A complex to trigger AMPK T172 dephosphorylation. Thus, UHRF1 serves as a bridging factor between PP2A and AMPK, thereby shutting off AMPK activity beyond its role in epigenetic and DNA damage regulation. The authors further uncovered that UHRF1 orchestrates glucose and lipid metabolism through suppressing AMPK activity. Notably, the interaction between AMPK and UHRF1 is dampened under metabolic stress, hence allowing for AMPK activation. Moreover, nuclear UHRF1 could also shut off AMPK activity and/or prevent AMPK activation by sequestering cytosolic AMPK in the nucleus (Fig. 1), although the mechanism underlying AMPK nuclear translocation by UHRF1 remains unclear. Collectively, these findings not only offer novel insight into how AMPK activity is terminated in the nucleus, but also expand the new role of UHRF1 as an adapter beyond its E3 ligase activity and epigenetic regulation.

To validate the role of UHRF1 in regulating AMPK activity and its associated biological outcomes in vivo, the authors have generated a transgenic mouse model where UHRF1 is conditionally overexpressed in mouse liver. Consistently, the mice with liverspecific UHRF1 overexpression display reduced AMPK activity in vivo, correlated with elevated serum glucose levels and triglyceride contents, insulin resistance, and lipid deposition in liver. Importantly, in liver-specific AMPKa1/a2 knockout (AMPKaLKO) mouse, liver-specific overexpression of UHRF1 through the adenovirus-delivery system failed to do so. Collectively, these in vivo studies underscore the novel role of UHRF1 in regulating glucose and lipid metabolism through AMPK suppression.

\footnotetext{
${ }^{1}$ Department of Cancer Biology, Wake Forest Baptist Medical Center, Wake Forest University, Winston-Salem, NC, USA. ${ }^{251}$ These authors contributed equally: Che-Chia Hsu,
}

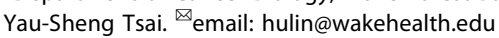




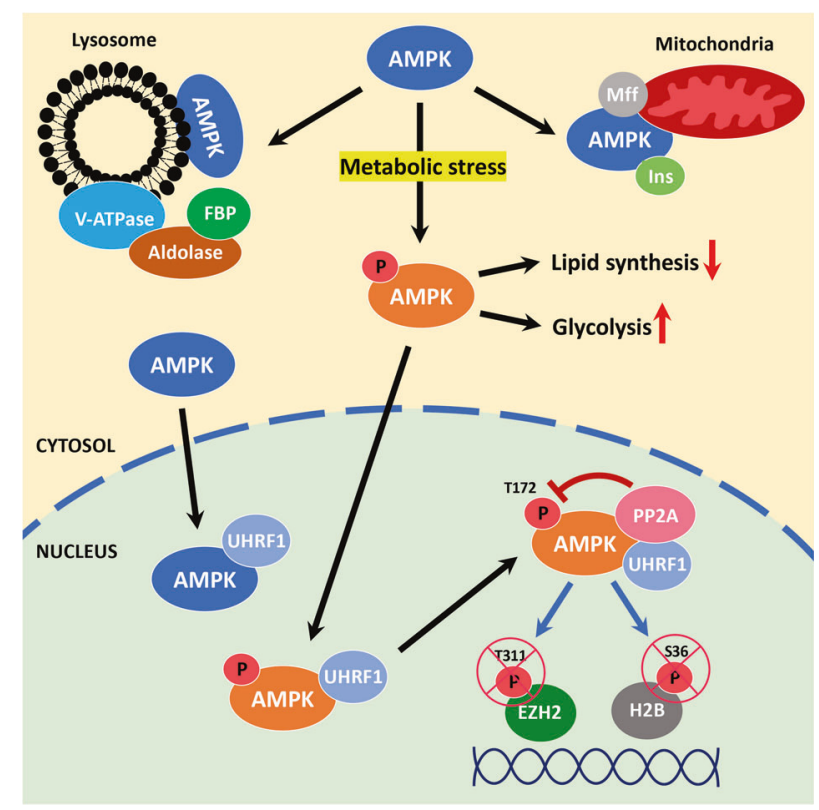

Fig. 1 Model of nuclear AMPK inhibition by UHRF1 regulating glucose and lipid metabolism. AMPK can be localized in the cytosol, lysosome, mitochondria and nucleus. AMPK is inactivated by FBP in lysosome and by inositol in mitochondria through distinct mechanisms. Nuclear UHRF1 sequesters cytosolic AMPK into the nucleus to shut off AMPK activity and/or prevent AMPK activation by serving as an adapter to recruit PP2A upon energy stress or bioenergetic failure. Inactivation of AMPK by UHRF1 blocks the AMPK-mediated phosphorylation of EZH2 at T311 and $\mathrm{H} 2 \mathrm{~B}$ at S36, and inhibit lipolysis and glycolysis. Inactive AMPK is indicated by color blue and active AMPK is indicated by color orange.

While this study has provided substantial advances in further understanding AMPK regulation, several important questions derived from this study remain to be addressed. First, while UHRF1 overexpression in liver drives insulin resistance in mice, it is unclear whether this regulation requires UHRF1's E3 ligase activity. This may offer the premise for targeting UHRF1 as a new strategy in the treatment of metabolic diseases. Second, since AMPK acts as a double-edged sword for cancer suppression or promotion, it is interesting to explore the functional crosstalk between UHRF1 and AMPK in cancer regulation. Third, although UHRF1 serves as a new guardian to maintain energy homeostasis by modulating AMPK activity, whether UHRF1 is regulated under the physiological or pathological conditions is also unknown. Fourth, since small portions of UHRF1 could be detected in cytoplasm, this raises the possibility that UHRF1 may recruit PP2A complex to shut off AMPK activity in cytoplasm. Finally, because AMPK activity is critically regulated by AMP/inositol and AMP/ATP ratios, it is important to explore whether UHRF1 may impact the levels of these metabolites thereby controlling AMPK activity. Addressing these key questions not only provide new insights into AMPK signaling regulation, but also offer new paradigms/strategies for targeting cancer and metabolic syndromes with AMPK dysregulation. Undoubtedly, this study opens a new avenue for AMPK research and may guide a new strategy for managing some human diseases.

\section{REFERENCES}

1. Hsu C. C., Peng D., Cai Z., Lin H. K. Semin. Cancer Biol. https://doi.org/10.1016/j. semcancer.2021.04.006 (2021)

2. Zhang, C. S. et al. Nature 548, 112-116 (2017).

3. Hsu, C. C. et al. Mol. Cell 81, 3803-3819 (2021).

4. Chauhan, A. S., Zhuang, L. \& Gan, B. Crit. Rev. Biochem. Mol. Biol. 55, 17-32 (2020).

5. Suzuki, A. et al. Mol. Cell. Biol. 27, 4317-4327 (2007).

6. Xu, X. et al. Cell Res. https://doi.org/10.1038/s41422-021-00565-y (2021).

7. Mudbhary, R. et al. Cancer Cell 25, 196-209 (2014).

8. Jia, Y. et al. Cell Discov. 2, 16007 (2016)

9. Bostick, M. et al. Science 317, 1760-1764 (2007).

10. Tian, Y. et al. Cell Rep. 10, 1957-1966 (2015).

\section{COMPETING INTERESTS}

The authors declare no competing interests.

\section{ADDITIONAL INFORMATION}

Correspondence and requests for materials should be addressed to Hui-Kuan Lin.

Reprints and permission information is available at http://www.nature.com/ reprints 